\title{
On Efficient Coloring of Chordless Graphs
}

\author{
Robert Janczewski, Michał Małafiejski
}

\begin{abstract}
We are given a simple graph $G=(V, E)$. Any edge $e \in E$ is a chord in a path $P \subseteq G$ (cycle $C \subseteq G$ ) iff a graph obtained by joining $e$ to path $P$ (cycle $C$ ) has exactly two vertices of degree 3 . A class of graphs without any chord in paths (cycles) we call pathchordless (cycle-chordless). We will prove that recognizing and coloring of these graphs can be done in $O\left(n^{2}\right)$ and $O(n)$ time, respectively. Our study was motivated by a wide range of applications of the graph coloring problem in coding theory, time tabling and scheduling, frequency assignment, register allocation and many other areas.
\end{abstract}

Keywords: vertex-coloring, chordless graphs, chromatic number

Mathematics Subject Classification: 05C15

Revised: 6 August 2009

\section{INTRODUCTION}

In the paper we study the problem of coloring of a special class of graphs, namely path- and cycle-chordless graphs, what we define in the later. The problem of graph coloring has many interesting applications in many areas: scheduling and timetabling, frequency assignment, register allocation, to mention only a few.

For example, let us sketch the application of the graph coloring in the register allocation problem. In this problem we have to assign variables to a limited number of hardware registers during the program execution, because variables located in the registers can be accessed much quicker than the variables outside registers. The allocation of variables should fulfill the following conditions: there are no conflicting variables, i.e. used by the same part of a program, and the size of the memory (outside regiters) should be minimized. To solve this problem we create a graph with nodes representing variables and the edges between conflicting nodes (variables). Now, it suffices to color this graph with a minimum number of colors, which is equivalent to the condition stated above, since the colors correspond to registers.

Let $G=(V, E)$ be a simple graph with vertex set $V=V(G)$ and edge set $E=E(G)$. By $n$ and $m$ we mean the number of vertices and the number of edges

\footnotetext{
* Department of Algorithms and System Modelling, Gdańsk University of Technology, Poland. E-mail: skalar,mima@eti.pg.gda.pl
} 
of $G$, respectively. By $\Delta(G)$ we denote the maximum degree over all vertices of graph $G$. Given $W \subset V$ by $G[W]$ we mean a subgraph of $G$ induced by $W$. A (closed) neighbourhood of $v \in V$ we denote by $N(v)(N[v])$.

By a chord in a path $P \subseteq G$ (cycle $C \subseteq G$ ) we mean any edge $e \in E(G)$ such that a graph obtained by joining $e$ to path $P$ (cycle $C$ ) has exactly two vertices of degree 3 . Our definition of a chord in a path is slightly different from that proposed in 1 , where a chord of a path $\left(v_{1}, \ldots, v_{k}\right)$ is any edge between two vertices of the path that is not an edge of the path.

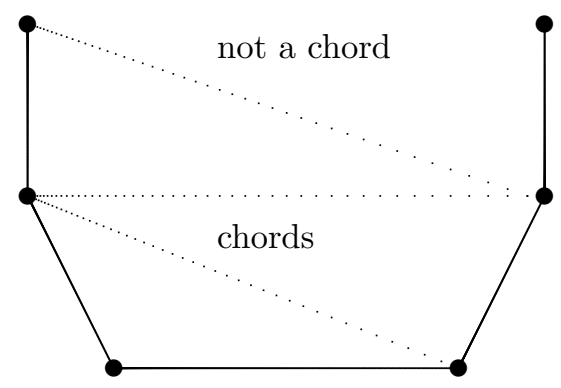

Fig. 1. An illustration of the definition of a chord in a path

The following observations are simple consequences of the definition:

- an edge $e \in E$ is a chord in path $P$ iff $e \notin E(P)$ is incident to two non-pendant vertices from $V(P)$,

- an edge $e \in E$ is a chord in cycle $C$ iff $e \notin E(C)$ is incident to two vertices from $V(C)$,

- vertices incident to any chord are of degree at least 3,

- any bridge cannot be a chord,

- any chord in a cycle with at least 5 vertices is a chord in a path.

A graph $G$ we call a path-chordless (cycle-chordless) if and only if there is no chords in any path (any cycle). The cycle-chordless graphs we will call shortly chordless graphs.

The following class of graphs are chordless:

- trees and cycles,

- cacti graphs, because any chord must belong to two cycles,

- graphs with the property: one of any adjacent vertices has degree at most 2, because any chord joins two vertices of degree at least 3,

- graphs with all cycle lengths divisible by $p>2$.

The following observations are immediate properties of the (path-) chordless graphs:

- $G$ is chordless iff any subgraph induced by a cycle of $G$ is a cycle,

- any subgraph of (path-) chordless graph is a (path-) chordless graph.

Despite the similiar definition of the path- and cycle-chordless graphs, these classes are indeed different. Two counterexample graphs are shown in Figure 2. 

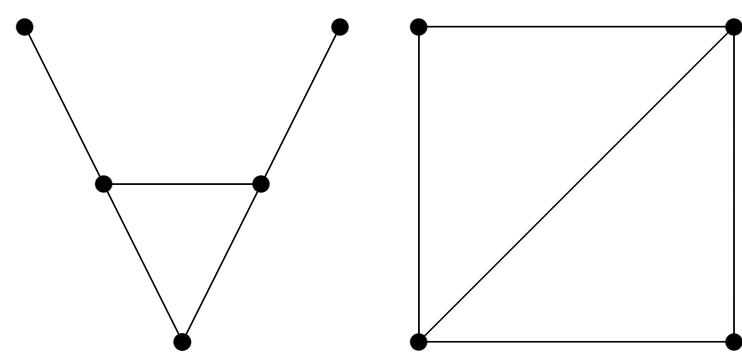

Fig. 2. The chordless graph and the path-chordless graph

\section{2. (PATH-) CHORDLESS GRAPHS AND OTHER CLASSES}

We will try to characterize the relations between chordless graphs and other classes, e.g. partial $k$-trees and $F$-free graphs. The class of chordless graphs seems to be different from known and well-described classes (see 1).

Proposition 1 For any $k>0$ there exists a (path-) chordless graph that is not

(1) almost $k$-tree,

(2) partial $k$-tree.

PROOF. (1) For any $k>0$ graph $K_{2, k+2}$ is $(k+4)$-vertex 2-connected (path-) chordless graph with $2 k+4$ edges. On the other hand, any 2 -connected almost $k$-tree has at most $2 k+3$ edges.

(2) Any graph such that no two vertices of degree at least 3 are adjacent is a (path-) chordless graph. Let $H^{\prime}$ be a $(k+2)$-vertex multigraph without loops such that any two vertices are connected with exactly $k+1$ edges. We obtain graph $H$ by putting an unique vertex on each edge. Graph $H$ is (path-) chordless graph and is not a partial $k$ tree. Conversely, suppose that $H$ is a partial $k$-tree. There exists a $k$-tree $G$ such that $H$ is its spanning subgraph. From definition of $k$-tree we can form a sequence $v_{1}, \ldots, v_{n}$ of all vertices of $G$ such that: $v_{1}, \ldots, v_{k}$ induce a clique and for every $i \geqslant k+1$ a subgraph $G\left[N\left[v_{i}\right] \cap\left\{v_{1}, \ldots, v_{i}\right\}\right]$ is a clique and $\left|N\left(v_{i}\right) \cap\left\{v_{1}, \ldots, v_{i}\right\}\right|=k$. Because $G\left[V\left(H^{\prime}\right)\right]$ is not a clique, so there exist $v_{i_{1}}, v_{i_{2}} \in V\left(H^{\prime}\right)$ non-adjacent in $G$. By definition of $H$ and $G$ there are at least $k+1$ vertices $v_{j_{1}}, \ldots, v_{j_{k+1}}$ adjacent to both $v_{i_{1}}$ and $v_{i_{2}}$ in graph $G$. There are two cases:

(1) $j_{l}:=\max \left\{j_{1}, \ldots, j_{k+1}\right\}>\max \left\{i_{1}, i_{2}\right\}$

Because $N\left[v_{j_{l}}\right] \cap\left\{v_{1}, \ldots, v_{j_{l}}\right\}$ is a clique in $G$ and $v_{i_{1}}, v_{i_{2}} \in N\left[v_{j_{l}}\right] \cap\left\{v_{1}, \ldots, v_{j_{l}}\right\}$, so $v_{i_{1}}$ is adjacent to $v_{i_{2}}$ in $G$, a contradiction.

(2) $i_{l}:=\max \left\{i_{1}, i_{2}\right\}>\max \left\{j_{1}, \ldots, j_{k+1}\right\}$

$\left|N\left(v_{i_{l}}\right) \cap\left\{v_{1}, \ldots, v_{i_{l}}\right\}\right|=k+1$, a contradiction. 
Let $F$ be a family of graphs. A graph $G$ is called $F$-free if $G$ contains no induced subgraph isomorphic to any member of $F$.

Proposition 2 cacti $\subset$ chordless $\subset K_{4}$-free.

Given any finite family $F$ including (path-) chordless graphs, there must be an $F$-free graph that is not (path-) chordless. We need the following

Proposition 3 Let $F$ be a family of graphs and let $G$ be not $F$-free graph such that every proper subgraph of $G$ is an $F$-free graph. Then graph $G$ is isomorphic to a certain member of $F$.

Theorem 1 If the class of all (path-) chordless graphs is equal to the class F-free graphs, then $F$ is infinite.

PROOF. Conversely, suppose that $F$ is a finite family of graphs, so $n:=$ $\max \{n(G): G \in F\}+5$ is properly defined. Moreover, by Proposition 3 a graph $G_{F}$ obtained from a cycle $C_{n}$ (path $P_{n}$ ) by joining a chord is isomorphic to a member of $F$, a contradiction.

\section{RECOGNIZING AND COLORING OF (PATH-)CHORDLESS GRAPHS}

We will prove that recognizing and coloring of (path-) chordless graphs can be done in polynomial time $\left(O\left(n^{2}\right)\right)$. Moreover, the coloring number ${ }^{1}$ of any connected (path-) chordless graph with $n \geqslant 5$ is not greater than 3 .

\subsection{COLORING OF (PATH-) CHORDLESS GRAPHS}

We need some special transformation of graph $G$. Let $U$ be any nonempty subset of $V(G)$ and let $u \neq v \in V \backslash U$. By $G(U ; u, v)$ we denote a graph obtained from $G$ by replacing all vertices from $U$ and its incident edges with a single vertex $w$ adjacent to $u$ and $v$ (see Fig. 3).

It is easy to see that this $U$-transformation does not change the chordless property of a graph.

Proposition 4 Let $G$ be any chordless graph, $U$ be any nonempty subset of $V(G)$ and let $u \neq v$ be vertices of $G$ non belonging to $U$. If there is a path $P \subset G[U \cup\{u, v\}]$ joining $u$ with $v$, then $G(U ; u, v)$ is a chordless graph.

Now, we will prove the essential theorem of this paper.

Theorem 2 If $n \geqslant 4$, then every $n$-vertex chordless graph has at least 2 non-adjacent vertices of degree at most 2.

\footnotetext{
1 The coloring number of a graph $G$, denoted $\operatorname{col}(G)$, is the smallest integer $d$ such that every subgraph of $G$ has a vertex with degree less than $d$.
} 


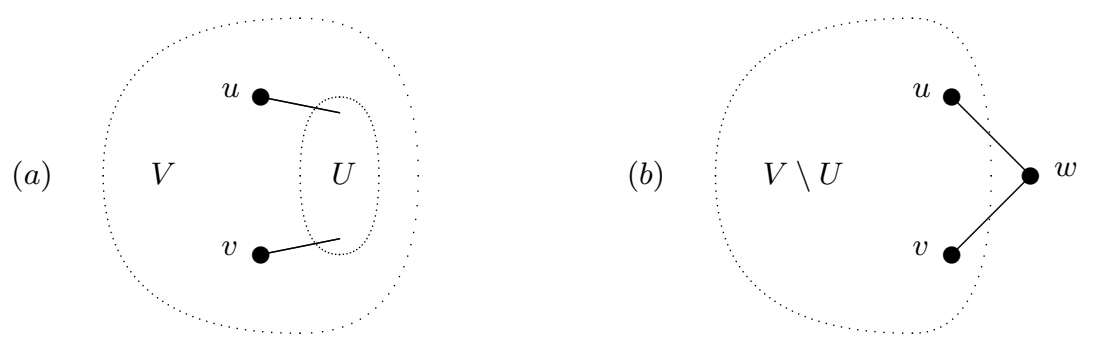

Fig. 3. (a) graph $G$; (b) graph $G(U ; u, v)$

PROOF. Suppose conversely, that there exists a graph $G$ with $n \geqslant 4$ vertices such that either it has at most one vertex of degree less than 3 or every two vertices of degree at most 2 are adjacent. Suppose, that $G$ is the smallest such graph, i.e. every graph $G^{\prime}$ with $n^{\prime}<n$ vertices has the desired property. It follows at once that $G$ is connected and has no pendant vertex. So, let $C \subset G$ be the shortest cycle in $G$. There are only three possibilities:

(1) Graph $G$ has at least 2 vertices of degree at most 2.

Because every two vertices of degree 2 are adjacent, so there are at most three such vertices only. Hence we have two possibilites:

(a) Graph $G$ has 2 vertices $u_{1}, u_{2}$ of degree 2 .

Let $N\left(u_{1}\right)=\left\{u_{2}, v_{1}\right\}$ and $N\left(u_{2}\right)=\left\{u_{1}, v_{2}\right\}$. If $v_{1}=v_{2}$ then graph $G \backslash\left\{u_{2}\right\}$ is a chordless graph with $n-1$ vertices, a contradiction. Otherwise, graph $G\left(\left\{u_{1}, u_{2}\right\} ; v_{1}, v_{2}\right)$ with $n-1$ vertices is a chordless graph with only one vertex of degree at most 2 , a contradiction.

(b) Graph $G$ has 3 vertices $u_{1}, u_{2}, u_{3}$ of degree 2 .

These three vertices form a triangle, so $G$ is disconnected, a contradiction.

(2) Graph $G$ has at most 1 vertex of degree 2 and there are two vertices $u_{1}$ and $u_{2}$ of cycle $C$ and one vertex $v_{1} \notin V(C)$ adjacent to both $u_{1}$ and $u_{2}$.

It is obvious that cycle $C$ cannot be a triangle. Moreover, $n(C)=4$, because otherwise graph $G\left[C \cup\left\{v_{1}\right\}\right]$ is a composition of two cycles $C^{\prime}$ and $C^{\prime \prime}$ (see Fig. 4), and one of them is shorter than $C$, a contradiction.

Let $N\left(u_{1}\right) \cap N\left(u_{2}\right)=\left\{v_{1}, \ldots, v_{s}\right\}$ and let $V\left(G_{i}\right) \ni v_{i}$ be a connected component of graph $G^{\prime}$ obtained from graph $G$ by deleting all edges $\left\{u_{j}, v_{r}\right\}$ for $j=1,2$ and $r=1, \ldots, s$. From this assumption it follows that $s \geqslant 3$. Now, observe that $G_{i} \neq G_{j}$, whenever $i \neq j$. Otherwise, $G_{i}=G_{j}$ for some $i \neq j$ and there is a path $P$ (not containing $u_{2}$ nor $v_{k}$ ) joining $v_{i}$ and $v_{j}$ in $G_{i}$, thus $\left\{u_{1}, v_{i}\right\}$ is a chord in $G$, which is shown in Fig. 5 .

There is at most one vertex $x$ of degree 2 in $G$, so let $G_{i}$ be a component of $G^{\prime}$ not containing $x$. If $\operatorname{deg}_{G}\left(v_{i}\right)>2$, then graph $G_{i}$ is a chordless graph with at most one vertex of degree less than 3 , with the number of vertices at least 4 , and smaller than $G$, a contradiction. Because $s \geqslant 3$, so there are at least 2 vertices $v_{i}$ and $v_{j}$ of degree 2 , a contradiction. 


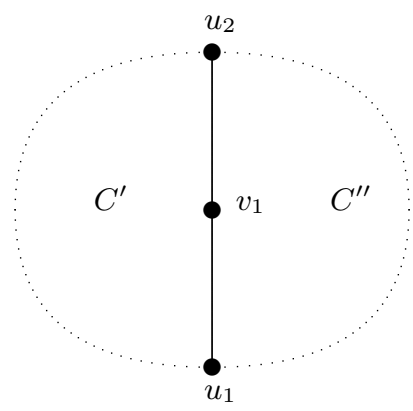

Fig. 4. Decompostion of the shortest cycle $C$

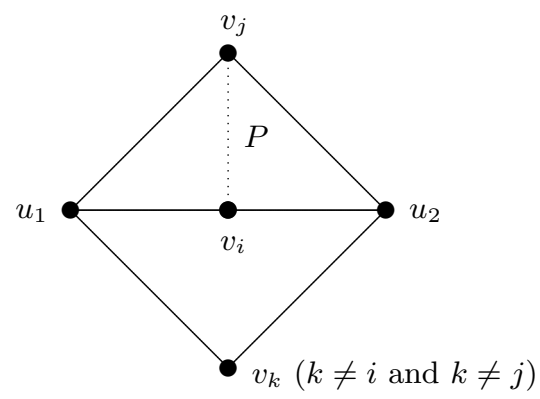

Fig. 5. The equality $G_{i}=G_{j}$ for $i \neq j$ implies the existance of a chord

(3) Graph $G$ has at most 1 vertex of degree 2 and for every two vertices $u_{1}$ and $u_{2}$ of cycle $C$ we have $N\left(u_{1}\right) \cap N\left(u_{2}\right) \subset V(C)$.

It is easy to see that for every vertex $x$ not belonging to $C$ there is at most one vertex from $C$ adjacent to $x$. Let $H$ be the graph obtained from $G$ by replacing all vertices from $C$ by a vertex $w$ and let us join vertex $w$ with all vertices from $G$ that has a neighbor in $C$. If $e=\{u, w\}$ is an edge in $H$, then $e^{*}=\{u, v\}$, where $v$ is the only one vertex from $C$ adjacent to $u$. There are only two possibilities:

(a) $H$ is a chordless graph.

It is clear that $n(H) \geqslant 4$. Because $n(H)<n(G)$ and all vertices common to $G$ and $H$ have the same degrees, so vertex $w$ is of degree at most 2 and for every $v \in V(C)$ we have $\operatorname{deg} v>2$. Thus we get $2 \geqslant \operatorname{deg}_{H}(w)=\sum_{v \in V(C)}\left(\operatorname{deg}_{G}(v)-\right.$ $2)) \geqslant n(C) \geqslant 3$, a contradiction.

(b) $H$ is not a chordless graph and some edge $e$ is a chord.

It is clear that $e$ is incident to $w$, because otherwise $e$ is a chord in a chordless graph $G$. Subgraph of $H$ containing cycle with a chord $e$ is shown in Fig. 6 . There are only two possible subgraphs of $G$ shown in Fig. 7: $(a)$ if $g_{1}^{*}$ and $g_{2}^{*}$ are not adjacent in $G,(b) g_{1}^{*}$ and $g_{2}^{*}$ are adjacent in $G$.

It is easy to see that $e^{*}$ is a chord in a graph shown in Fig. $7(a)$. Consider graph shown in Fig. $7(b)$. Assume that there is a path joining some vertex from cycle $C$ with a vertex from $P^{\prime}$ or $P^{\prime \prime}$ and not containing $e^{*}$ and $u$. 


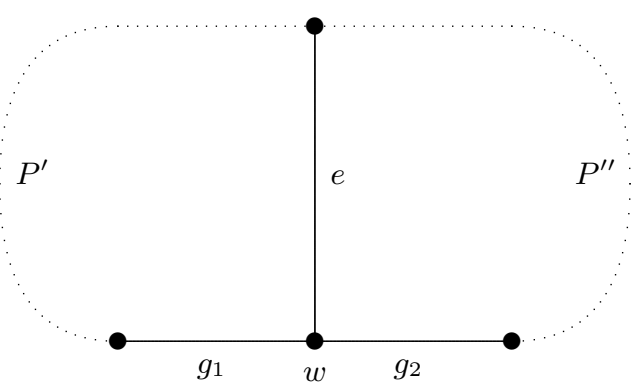

Fig. 6. A subgraph of $H$ containing cycle with a chord $e$

(a) $: P^{\prime}$

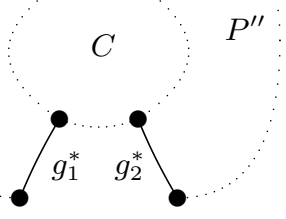

(b) $: P^{\prime}$

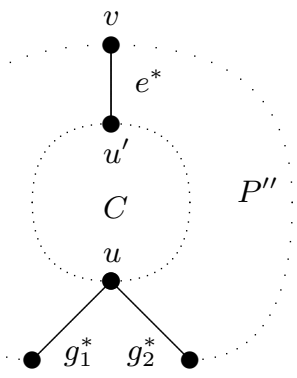

Fig. 7. Two possible subgraphs of $G$

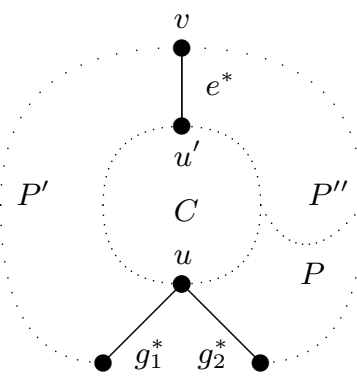

Fig. 8. The shortest path $P$ joining cycle $C$ with one of the paths $P^{\prime}$ or $P^{\prime \prime}$

Let $P$ be the shortest such path, shown in Fig. 8 . It is easy to see that $e^{*}$ is a chord.

Let $V^{\prime}$ be the set of vertices of the component of graph $\left(V \backslash\{u\}, E \backslash\left\{e^{*}\right\}\right)$ containing all vertices of $C$. There are only two possibilities:

i. The only one vertex of degree 2 in $G$ is in $V^{\prime}$.

Graph $G^{\prime}=G\left(V^{\prime} ; u, v\right)$ is chordless and $n\left(G^{\prime}\right) \geqslant 4$. Thus we get that all vertices common to $G$ and $G^{\prime}$ have the same degree (besides $u$ ) and $\operatorname{deg}_{G^{\prime}}(u) \geqslant 3$, so $G^{\prime}$ has only one vertex of degree at most 2 , a contradiction. 
ii. All vertices from $V^{\prime}$ have degree $\geqslant 3$ in $G$.

Graph $G^{\prime \prime}=G\left(V \backslash\left(V^{\prime} \cup\{u\}\right) ; u^{\prime}, u\right)$ is a chordless graph and $n\left(G^{\prime \prime}\right) \geqslant 4$. Analogously, all vertices common to $G$ and $G^{\prime \prime}$ have the same degree (besides $u$ ) and $\operatorname{deg}_{G^{\prime \prime}}(u) \geqslant 3$, so there is only one vertex in $G^{\prime \prime}$ of degree at most 2 , a contradiction.

As a simple consequence of Theorem 2 we have a tight bound on the number of edges of any chordless graph.

Corollary 3 For any connected chordless graph with $n>3$ we have

$$
m \leqslant 2 n-4
$$

PROOF. One can check that for $n=4$ and $n=5$ the thesis obviously follows. Consider a chordless graph $G$ with $n+1$ vertices and assume that for every chordless graph with $n$ vertices the thesis follows. By Theorem 2 there are two non-adjacent vertices $u$ and $w$ of degree at most 2 . Let $G^{\prime}$ be the graph obtained from $G$ by removing the vertices $u$ and $w$ and incident edges. From the assumption it follows $m\left(G^{\prime}\right) \leqslant 2 n\left(G^{\prime}\right)-4$, hence $m(G) \leqslant m\left(G^{\prime}\right)+4 \leqslant 2\left(n\left(G^{\prime}\right)+2\right)-4=2 n(G)-4$.

The bound is tight. It suffices to consider complete bipartite graphs $K_{2, n-2}$.

\section{Corollary 4 chordless $\subset 2$-degenerated ${ }^{2}$.}

Corollary 5 Any chordless graph is 3-colorable.

Theorem 6 Let $n \geqslant 5$, any connected $n$-vertex path-chordless graph has at least two non-adjacent vertices of degree at most 2 .

PROOF. Let $G$ be $n$-vertex connected path-chordless graph. Because $K_{4}$ with an attached pendant vertex is the path-chordless graph, so $K_{4}$ is not a subgraph of $G$. If graph $G$ is a chordless graph, then by Theorem 2 the thesis follows. Suppose, that edge $e$ is a chord in some cycle $C \subset G$. It is easy to see that $n(C)=4$. Let $V(C)=\left\{v_{1}, v_{2}, v_{3}, v_{4}\right\}$ and $e=\left\{v_{1}, v_{3}\right\}$, which is shown in Fig. 9.

Obviously, $v_{2}$ and $v_{4}$ are not adjacent in $G$. If $v_{2}$ (or $v_{4}$ ) has a neighbor $u \notin V(C)$, then edge $\left\{v_{2}, v_{3}\right\}\left(\left\{v_{3}, v_{4}\right\}\right)$ is a chord in $G$, so $\operatorname{deg}_{G}\left(v_{2}\right)=\operatorname{deg}_{G}\left(v_{4}\right)=2$, and the proof is complete.

If a path-chordless graph $G$ has no vertex of degree $\leqslant 2$, then every connected component of $G$ is $K_{4}$.

Corollary 7 Every path-chordless graph is 4-colorable, and the chromatic number is equal to 4 iff one of the connected components is $K_{4}$.

Corollary 8 Let $G$ be a path-chordless graph with $n \geqslant 5$ vertices, then $m \leqslant 2 n-4$.

\footnotetext{
${ }^{2}$ Graph $G$ is $d$-degenerated if and only if $\operatorname{col}(G) \leqslant d+1$.
} 


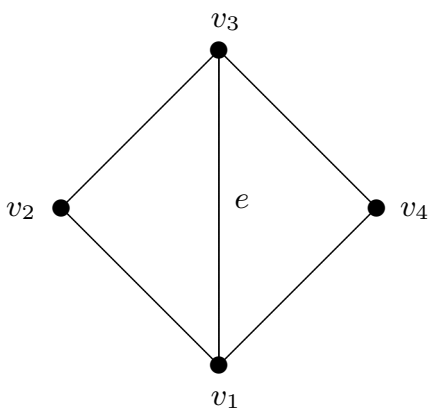

Fig. 9. Edge e is a chord in some cycle

Analogously, this bound is tight because $K_{2, n-2}$ is a path-chordless graph.

Summarizing, we have just proved that every (path-) chordless graph with $n \geqslant 5$ is 3-colorable and has $O(n)$ edges. This allows us to use the well-known algorithm for optimal coloring of bipartite graphs and the SL algorithm 2 to obtain the coloring with the minimal number of colors. Since both algorithms run in $O(m+n)$ time and for (path-) chordless graphs $m=O(n)$, we see that they can be optimally colored in linear time.

\subsection{RECOGNIZING OF THE (PATH-) CHORDLESS GRAPHS}

We wil prove that these graphs can be recognized in polynomial time. It suffices to check whether each edge is a chord or not.

Proposition 5 For a given graph $G$ and edge $e=\{u, v\}$ checking whether $e$ is a chord (in a cycle) can be done in $O(m+n)$ time.

PROOF. Let $H=G \backslash e$, it is easy to see that $e$ is a chord (in a cycle) in $G$ iff $u$ and $v$ are vertices of a cycle $C \subset H$, hence $e$ is a chord iff $u$ and $v$ are in the same 2-connected component of $H$, which can be done in $O(m+n)$ time (see 1).

Because the number of edges of any chordless graph is $O(n)$, we can identify all chords in a graph in $O\left(n^{2}\right)$ time.

Proposition 6 For a given graph $G$ and edge $e=\{u, v\}$ checking whether $e$ is a chord (in a path) can be done in $O(m+n)$ time.

PROOF. Let $H=G \backslash e$ and $A=N(u) \cap N(v), B=N(u) \backslash N(v)$ and $C=N(v) \backslash N(u)$ (in $H)$. Finding these sets can be done in $O(n)$ time. There are only four possibilities:

(1) $A \neq \emptyset, B \neq \emptyset, C \neq \emptyset$. Then $e$ is a chord in a path with endpoints in $B$ and $C$ and containing $u, v$ and a vertex from $A$.

(2) $A \neq \emptyset$ and $(B=\emptyset$ or $C=\emptyset$ ). Then $e$ is a chord (in a path) iff $B \cup C \neq \emptyset$ and $|A| \geqslant 2$. 
(3) $A=\emptyset$ and $(|B| \leqslant 1$ or $|C| \leqslant 1)$. Then $e$ is not a chord (in any path), because at least one of the vertices $u$ or $v$ is of degree at most 2 .

(4) $A=\emptyset,|B| \geqslant 2,|C| \geqslant 2$. Then $e$ is a chord in a path iff $u$ and $v$ are in the same connected component of $H$ (needs $O(m+n)$ time).

Analogously, because the number of edges of any path-chordless graph is $O(n)$, so we can identify all chords (in a path) in a graph in $O\left(n^{2}\right)$ time.

\section{REFERENCES}

Brandstädt, A., Le, V.B., Spinrad, J.P. (1999). Graph Classes: A Survey, SIAM Monographs on Discrete Mathematics and Applications, Philadelphia.

Matula, D.W., Marble, G., Isaacson, D. (1972). Graph coloring algorithms, in: Graph Theory and Computing, Academic Press, New York, pp. 109-122. 